\title{
GENERALIZED FLAG MANIFOLDS BOUND EQUIVARIANTLY
}

\author{
HARSH V. PITTIE
}

ABSTRACT. Given a compact, connected lie group $G$ and a maximal torus $T$, we give a simple, explicit construction of a $G$-manifold $M$ which bounds the homogeneous space $G / T$ equivariantly.

Let $G$ be a compact, connected lie group with a maximal torus $T$. We will construct a compact manifold $M$ with a $G$-action, and a $G$-equivariant imbedding $G / T \rightarrow M$ which identifies $G / T$ with the boundary of $M$. It is known [1] that the Pontryagin and Stiefel-Whitney classes of $G / T$ vanish, so certainly the corresponding characteristic numbers; and hence by general results of cobordism theory, $G / T$ bounds. However, this result about the characteristic classes requires a detailed study of the cohomology of $G / T$. In any case, the deduction from cobordism theory does not provide any "simple" explicit manifold bounding $G / T$, let alone equivariantly.

We start with the well-known decomposition for the lie algebra $g$ of $G$, as an oriented ad $(T)$-module: $g=t \oplus \Sigma_{a>0} g_{a}$, where $t$ is the lie algebra of $T$ and $g_{a}$ are irreducible, oriented ad(T)-planes corresponding to the (positive) roots $\alpha: t \rightarrow R$. The subspace $c_{a}$ generated by $t$ and $g_{\alpha}$ is actually a lie subalgebra $[2, \text { Chapter } 6]^{1}$ isomorphic to $a \oplus \operatorname{su}(2)$, where $a=\operatorname{Ker}(\alpha)$ is an abelian ideal, and $s u(2)$ is generated by the coroot $H_{a} \in t$ and $g_{a}$. Denoting by $C_{a} \subseteq G$ the connected subgroup corresponding to $C_{a}$, it is easy to see that $C_{a} / T \simeq S^{2}$, the two-sphere. Here is a quick proof: $C_{a} / T$ is a compact two-manifold, and since any compact lie group modulo its maximal torus is simply-connected, it must be $S^{2}$. Note that $S^{2}$ acquires a natural orientation from $g_{a}$.

Now consider the homogeneous fibre-bundle $C_{a} / T \rightarrow G / T \rightarrow G / C_{a}$. This exhibits $G / T$ as $G \times C_{\alpha}\left(S^{2}\right)$ as a $G$-space, with $G$ acting on the latter space by left multiplication in the first factor. Our main observation is that

Received by the editors January 3, 1974.

AMS (MOS) subject classifications (1970). Primary 57D85, 57E15.

Key words and phrases. Compact lie group, maximal torus, cobordism.

1 Serre [2] only discusses the semisimple case, but since $g$ is reductive, the same argument applies. 
the $C_{a^{-a c t i o n}}$ on $S^{2}$ is equivalent to one through $S O(3)$, hence extends to a $C_{\alpha}$-action on the three-disk $D^{3}$. This can be seen directly, if one writes out the isomorphism $C_{a} / T \simeq S^{2}$ using the lie theory above. For our purposes, we can invoke "uniformization" since $C_{\alpha}$ preserves some complex structure $[1, \S 12]$-or equivalently, some Riemannian metric-on $S^{2}$. Therefore we construct the manifold $M=G \times C_{a}\left(D^{3}\right)$, with $G$ acting on $M$ by left multiplication in the first factor. The obvious inclusion $G \times C_{a}\left(S^{2}\right) \rightarrow G \times C_{a}\left(D^{3}\right)$ then gives the required imbedding.

In a word, we have "filled in" the two-spheres in the fibre-bundle above. Note that there is a G-equivariant fibre-map $\pi: M \rightarrow G / C_{\alpha}$ with fibre $D^{3}$, and our homogeneous bundle above is the "boundary-bundle" of $D^{3} \rightarrow M \stackrel{\pi}{\rightarrow} G / C_{\alpha}$.

\section{B IB LIOG R A PHY}

1. A. Borel and F. Hirzebruch, Characteristic classes and homogeneous spaces, Amer. J. Math. 80 (1958), 458-538. MR 21 \#1586.

2. J.-P. Serre, Algèbres de Lie semi-simples complexes, Benjamin, New York, 1966. MR $35 \# 6721$.

COURANT INSTITUTE OF MATHEMATICAL SCIENCES, NEW YORK UNIVERSITY, NEW YORK, NEW YORK 10012 